\title{
A linear model of gravity wave drag for hydrostatic sheared flow over elliptical mountains
}

Article

Accepted Version

Teixeira, M. A. C. and Miranda, P. M. A. (2006) A linear model of gravity wave drag for hydrostatic sheared flow over elliptical mountains. Quarterly Journal of the Royal Meteorological Society, 132 (620). pp. 2439-2458. ISSN 1477-870X doi: https://doi.org/10.1256/qj.05.220 Available at https://centaur.reading.ac.uk/29248/

It is advisable to refer to the publisher's version if you intend to cite from the work. See Guidance on citing.

To link to this article DOI: http://dx.doi.org/10.1256/qj.05.220

Publisher: Royal Meteorological Society

All outputs in CentAUR are protected by Intellectual Property Rights law, including copyright law. Copyright and IPR is retained by the creators or other copyright holders. Terms and conditions for use of this material are defined in the End User Agreement.

www.reading.ac.uk/centaur 
Central Archive at the University of Reading

Reading's research outputs online 


\title{
A linear model of gravity wave drag for hydrostatic sheared flow over elliptical mountains
}

\author{
By M. A. C. Teixeira* and P. M. A. Miranda \\ University of Lisbon, CGUL, IDL, Lisbon, Portugal \\ (Received 1 January 2002; revised 31 January 2002)
}

SUMMARY

An analytical model of orographic gravity wave drag due to sheared flow past elliptical mountains is developed. The model extends the domain of applicability of the well-known Phillips model to wind profiles that vary relatively slowly in the vertical, so that they may be treated using a WKB approximation. The model illustrates how linear processes associated with wind profile shear and curvature affect the drag force exerted by the airflow on mountains, and how it is crucial to extend the WKB approximation to second order in the small perturbation parameter for these effects to be taken into account. For the simplest wind profiles, the normalised drag depends only on the Richardson number of the flow at the surface, $R i$, and on the aspect ratio of the mountain, $\gamma$. For a linear wind profile, the drag decreases as $R i$ decreases, and this variation is faster when the wind is across the mountain than when it is along the mountain. For a wind that rotates with height maintaining its magnitude, the drag generally increases as $R i$ decreases, by an amount depending on $\gamma$ and on the incidence angle. The results from WKB theory are compared with exact linear results and also with results from a non-hydrostatic nonlinear numerical model, showing in general encouraging agreement, down to values of $R i$ of order one.

KEYWORDS: Gravity wave drag, Linear theory, WKB approximation

\section{INTRODUCTION}

It is well known that mesoscale mountains have an important impact on the large-scale atmospheric circulation. As the stably stratified tropospheric air flows over these mountains, internal gravity waves are generated. The associated pressure perturbation induces a drag force that acts on the mountains. The gravity waves transport momentum upward, which is eventually deposited at critical layers, decelerating the large-scale flow. The dependence of gravity wave drag (GWD) on the background flow parameters is a problem of obvious fundamental importance, since the parameter space is very large, and by no means totally explored. It is also a problem of practical importance, since GWD must be parameterised in general circulation models (GCMs) and other largescale numerical models where the horizontal resolution is insufficient to explicitly represent these waves.

The drag on an isolated mountain depends on characteristics of the mountain, such as its height, width and horizontal aspect ratio, and on characteristics of the background flow, such as the wind velocity and the density stratification. Linear analytical models are especially useful for studying these dependences. While it is clear that the variation of the wind with height causes refraction of the gravity waves, and this has an impact on the drag, most analytical studies have considered wind profiles with constant velocity (Smith 1979, 1980; Phillips 1984), or at most a linear variation (Smith 1986, Keller 1994, Shutts 1995, Grubišić and Smolarkiewicz 1997), because these are the only cases where an exact analytical solution is straightforward. Teixeira et al. (2004) and Teixeira and Miranda (2004) developed a more general approach, where the Taylor-Goldstein equation is solved using the WKB approximation. This is formally valid for a wind that varies

\footnotetext{
* Corresponding author: Centro de Geofísica da Universidade de Lisboa, Edifício C8, Campo Grande, 1749-016 Lisbon, Portugal.

(c) Royal Meteorological Society, 2002.
} 
relatively slowly with height, but in practise was seen to be useful for flows with Richardson numbers of order one.

The WKB approximation amounts to assuming that the vertical wavenumber of the gravity waves is a slow function of height, and expanding it in a power series of a small parameter. This procedure has been followed previously by some authors (Grisogono 1994, Shutts and Gadian 1999), but the novelty in Teixeira et al. (2004) is that the power series is extended up to second order. This turns out to be crucial for the effect of the shear and curvature of the wind profile to have an impact on the drag. Teixeira et al. (2004) considered flow over an axisymmetric mountain, and Teixeira and Miranda (2004) treated the case of flow over a 2D ridge. In both cases, they found that, in the hydrostatic approximation, the drag for a sheared wind could be expressed as the drag in the absence of shear times a correction that does not depend on the detailed shape of the orography. Their model explained why in some sheared flows the drag decreases as the Richardson number decreases (Grubišić and Smolarkiewicz 1997) while in others there is an opposite trend (Valente 2000), and showed that the drag on an axisymmetric mountain may not be perfectly aligned with the surface wind.

For purposes of GWD parameterisation, it is important to consider flow over obstacles that are anisotropic but not 2D, of which mountains with an elliptical horizontal cross-section are the simplest example. This problem was addressed by Phillips (1984) for a constant background wind. Since some statistical GWD parameterisation schemes that are used operationally are partially adapted from Phillips' theory (Baines and Palmer 1990, Lott and Miller 1997), it is of great interest to extend this theory to the case of a sheared background wind. This study aims to do just that, by extending the model of Teixeira et al. (2004) to flow over elliptical mountains. As in the studies of Teixeira et al. (2004) and Teixeira and Miranda (2004), the expressions derived here give the GWD as the drag in the absence of shear times a correction due to the variation of the wind with height. This correction now depends on the ratio of the lengths of the main axes of the elliptical cross section of the mountain, but is otherwise independent of the detailed shape of the orography. The results to be presented are thus rather general and, hopefully, easy to implement in existing parameterisation schemes.

Whilst for practical aspects of GWD parameterisation, an analysis of nonlinear processes is essential, this study is limited in its scope to linear processes, which are complicated enough to deserve detailed attention, and are better understood in isolation from other flow complications.

This paper is organised as follows: in section 2 the analytical model used in this study is presented. In section 3, mountain wave drag is calculated, and its behaviour is exemplified for two simple flows. Finally, in section 4, the main conclusions are presented.

\section{HyDROSTATIC FLOW OVER GENTLE OROGRAPHY}

Following Phillips (1984), flow over an isolated elliptical mountain is considered. The inviscid, steady and non-rotating equations of motion with the Boussinesq approximation are linearised with respect to the perturbations induced by the mountain and the hydrostatic approximation is used (see also Smith 1980).

The Boussinesq approximation is justified when variations in density are important for the buoyancy but not for the inertia of the fluid (Gill 1982). The linear and hydrostatic approximations are formally valid when the dimensionless 
height of the mountain $N h_{0} /|\mathbf{U}| \ll 1$ and its dimensionless width $N a /|\mathbf{U}| \gg 1$ (where $h_{0}$ and $a$ are the height and half-width of the mountain, $|\mathbf{U}|$ is the magnitude of the background wind, and $N$ is the Brunt-Väisälä frequency). Note that these conditions imply that the slope of the mountain is very small. Despite these limitations, the linear approximation has the decisive feature of making an analytical treatment feasible, while the hydrostatic approximation is justified by scaling arguments using typical flow scales. For example, for $a=10 \mathrm{~km}, N=0.01 \mathrm{~s}^{-1}$ and $|\mathbf{U}|=10 \mathrm{~ms}^{-1}, N a /|\mathbf{U}|=10$. Additionally, it is known that most of the subgrid GWD in numerical models comes from waves that are approximately hydrostatic, because both rotation and non-hydrostatic effects decrease the drag (Smith 1979, Miranda and James 1992).

Since the background flow is assumed to be horizontally homogeneous, and an isolated mountain is considered, the terrain elevation $h(x, y)$ and all flow perturbations may be expressed as Fourier integrals along $x$ and $y$ (Smith 1980).

When the equations of motion are combined and expressed in Fourier space, a single equation for $\hat{w}$, the Fourier transform of the vertical velocity perturbation, is obtained,

$$
\hat{w}^{\prime \prime}+\left[\frac{N^{2} k_{12}^{2}}{\left(U k_{1}+V k_{2}\right)^{2}}-\frac{U^{\prime \prime} k_{1}+V^{\prime \prime} k_{2}}{U k_{1}+V k_{2}}\right] \hat{w}=0
$$

where the prime denotes differentiation with respect to $z,(U, V)$ is the (horizontal) wind vector, $\left(k_{1}, k_{2}\right)$ is the horizontal wavenumber vector, and $k_{12}=$ $\left(k_{1}^{2}+k_{2}^{2}\right)^{1 / 2}$. This, which is known as the Taylor-Goldstein equation, is subject to the boundary conditions that the flow follows the terrain at the surface,

$$
\hat{w}(z=0)=i \hat{h}\left[U(z=0) k_{1}+V(z=0) k_{2}\right]
$$

(where $\hat{h}$ is the Fourier transform of the terrain elevation) and that the energy of the internal gravity waves radiates upwards (the radiation boundary condition).

An approximate solution to (1) may be obtained using the WKB approximation (Bender and Orszag 1999). The vertical coordinate is first rescaled as $Z=\varepsilon z$, where $\varepsilon$ is a small dimensionless parameter, with the consequence that $\partial / \partial z=\varepsilon \partial / \partial Z$. This enables (1) to be expressed as

$$
\varepsilon^{2} \ddot{\hat{w}}+\left[\frac{N^{2} k_{12}^{2}}{\left(U k_{1}+V k_{2}\right)^{2}}-\varepsilon^{2} \frac{\ddot{U} k_{1}+\ddot{V} k_{2}}{U k_{1}+V k_{2}}\right] \hat{w}=0,
$$

where differentiation with respect to $Z$ has been replaced by a dot.

The approximate WKB solution to (3) valid up to second order in $\varepsilon$ (Teixeira et al. 2004) is

$$
\hat{w}(Z)=\hat{w}(Z=0) \exp \left[i \varepsilon^{-1} \int_{0}^{Z}\left(m_{0}(\zeta)+\varepsilon m_{1}(\zeta)+\varepsilon^{2} m_{2}(\zeta)\right) d \zeta\right],
$$

where the vertical wavenumber of the waves, $m=m_{0}+\varepsilon m_{1}+\varepsilon^{2} m_{2}+\ldots$, is assumed to be a slow function of $z$ and is expanded in a power series of $\varepsilon$. When (4) is introduced into (3), three equations are obtained for $m_{0}, m_{1}$ and $m_{2}$, which may be solved successively, at zeroth, first and second-order in $\varepsilon$, yielding the 
following solutions:

$$
\begin{aligned}
m_{0} & =\frac{N k_{12}}{U k_{1}+V k_{2}}, \\
m_{1} & =-\frac{1}{2} i \frac{\dot{U} k_{1}+\dot{V} k_{2}}{U k_{1}+V k_{2}}, \\
m_{2} & =-\frac{1}{8} \frac{U k_{1}+V k_{2}}{N k_{12}}\left[\left(\frac{\dot{U} k_{1}+\dot{V} k_{2}}{U k_{1}+V k_{2}}\right)^{2}+2 \frac{\ddot{U} k_{1}+\ddot{V} k_{2}}{U k_{1}+V k_{2}}\right],
\end{aligned}
$$

where it was assumed that $N$ is constant. Together with (2), (5)-(7) completely define the solution to the internal gravity wave problem, (4). Note that the sign of $m_{0}$ has been chosen so that it is the same as that of $\left(U k_{1}+V k_{2}\right)$, in order to satisfy the radiation boundary condition.

Combining the momentum equations in Fourier space yields an expression for the Fourier transform of the pressure perturbation, which is given by

$$
\hat{p}=i \frac{\rho_{0}}{k_{12}^{2}}\left[\left(U^{\prime} k_{1}+V^{\prime} k_{2}\right) \hat{w}-\left(U k_{1}+V k_{2}\right) \hat{w}^{\prime}\right],
$$

where $\rho_{0}$ is a reference density (assumed to be constant). When expressed in terms of the scaled vertical coordinate and $m_{0}, m_{1}$ and $m_{2}$, this becomes:

$$
\hat{p}=i \frac{\rho_{0}}{k_{12}^{2}}\left[\varepsilon\left(\dot{U} k_{1}+\dot{V} k_{2}\right)-i\left(U k_{1}+V k_{2}\right)\left(m_{0}+\varepsilon m_{1}+\varepsilon^{2} m_{2}\right)\right] \hat{w}
$$

where the fact that $\dot{\hat{w}}=i \varepsilon^{-1}\left(m_{0}+\varepsilon m_{1}+\varepsilon^{2} m_{2}\right) \hat{w}$ (which results from (4)) has been used. It may be noted from (9) that $\hat{p}$ is expressed as a power series of $\varepsilon$. In particular, if at the surface

$$
\hat{p}(z=0)=\hat{p}_{0}+\varepsilon \hat{p}_{1}+\varepsilon^{2} \hat{p}_{2},
$$

when the definitions (5)-(7) and (2) are used in (9), it is found that

$$
\begin{aligned}
& \hat{p}_{0}=i \frac{\rho_{0} N}{k_{12}}\left(U_{0} k_{1}+V_{0} k_{2}\right) \hat{h} \\
& \hat{p}_{1}=-\frac{1}{2} \frac{\rho_{0}}{k_{12}^{2}}\left(U_{0} k_{1}+V_{0} k_{2}\right)\left(\dot{U}_{0} k_{1}+\dot{V}_{0} k_{2}\right) \hat{h} \\
& \hat{p}_{2}=-\frac{1}{8} i \frac{\rho_{0}}{k_{12}^{3}} \frac{\left(U_{0} k_{1}+V_{0} k_{2}\right)^{3}}{N}\left[\left(\frac{\dot{U}_{0} k_{1}+\dot{V}_{0} k_{2}}{U_{0} k_{1}+V_{0} k_{2}}\right)^{2}+2 \frac{\ddot{U}_{0} k_{1}+\ddot{V}_{0} k_{2}}{U_{0} k_{1}+V_{0} k_{2}}\right] \hat{h},
\end{aligned}
$$

where $\left(U_{0}, V_{0}\right)=(U(z=0), V(z=0))$ is the background wind velocity at the surface.

\section{MOUNTAin WAVE DRAG}

In the linear approximation, the drag force exerted on a mountain is in general given by

$$
\left(D_{x}, D_{y}\right)=\int_{-\infty}^{+\infty} \int_{-\infty}^{+\infty} p(z=0)\left(\frac{\partial h}{\partial x}, \frac{\partial h}{\partial y}\right) d x d y
$$


where $p$ is the pressure perturbation. This may be expressed in Fourier space as

$$
\left(D_{x}, D_{y}\right)=4 \pi^{2} i \int_{-\infty}^{+\infty} \int_{-\infty}^{+\infty}\left(k_{1}, k_{2}\right) \hat{p}^{*}(z=0) \hat{h} d k_{1} d k_{2}
$$

where the asterisk denotes complex conjugate. It is clear from this definition and from (10) that the drag itself is also given by a power series of $\varepsilon$,

$$
\mathbf{D}=\mathbf{D}_{0}+\varepsilon \mathbf{D}_{1}+\varepsilon^{2} \mathbf{D}_{2}
$$

where $\mathbf{D}=\left(D_{x}, D_{y}\right)$ and

$$
\mathbf{D}_{j}=4 \pi^{2} i \int_{-\infty}^{+\infty} \int_{-\infty}^{+\infty} \mathbf{k} \hat{p}_{j}^{*} \hat{h} d k_{1} d k_{2}, \quad j=0,1,2,
$$

with $\mathbf{k}=\left(k_{1}, k_{2}\right)$.

In order to simplify the drag calculation, the fact that the mountain under consideration has an elliptical horizontal cross section must be taken into account. The terrain elevation of such a mountain is given by $h(x, y)=h(r)$, where $r=\left[(x / a)^{2}+(y / b)^{2}\right]^{1 / 2}$ and $a$ and $b$ are the half-widths of the mountain along $x$ and $y$ (the main axes of the ellipse). As a consequence, the Fourier transform of the terrain elevation $\hat{h}$ is only a function of $\rho=\left(a^{2} k_{1}^{2}+b^{2} k_{2}^{2}\right)^{1 / 2}$. This property suggests the introduction of elliptical polar coordinates for the evaluation of the integrals in (17). The Cartesian wavenumber coordinates are defined in terms of their elliptical counterparts as

$$
k_{1}=\frac{\rho}{a} \cos \theta, \quad k_{2}=\frac{\rho}{b} \sin \theta .
$$

When (11)-(13) are introduced into (17) and (18) is used, the integrations over $\rho$ and over $\theta$ may be separated. The zeroth-order drag then takes the form

$$
D_{0 x}=\rho_{0} N U_{0} b h_{0}^{2} G B(\gamma), \quad D_{0 y}=\rho_{0} N V_{0} b h_{0}^{2} G C(\gamma),
$$

where

$$
\begin{aligned}
G & =16 \pi^{2} \int_{0}^{+\infty} \rho^{2}\left|\hat{h}^{\prime}\right|^{2} d \rho, \\
B(\gamma) & =\int_{0}^{\pi / 2} \frac{\cos ^{2} \theta}{\left(\cos ^{2} \theta+\gamma^{2} \sin ^{2} \theta\right)^{1 / 2}} d \theta, \\
C(\gamma) & =\gamma^{2} \int_{0}^{\pi / 2} \frac{\sin ^{2} \theta}{\left(\cos ^{2} \theta+\gamma^{2} \sin ^{2} \theta\right)^{1 / 2}} d \theta .
\end{aligned}
$$

$\hat{h}^{\prime}=\hat{h} /\left(h_{0} a b\right)$ is the dimensionless Fourier transform of the terrain elevation and $\gamma=a / b$ is the horizontal aspect ratio of the mountain. The coefficients $G, B$ and $C$ defined in (20)-(22) are the same as derived in Phillips (1984). $G$ encapsulates the variation of the drag with the terrain shape in the radial direction. For the present purposes, and unlike what was done in Phillips (1984), an explicit functional form for $\hat{h}^{\prime}$ will not be specified, so $G$ keeps the generic form (20), unless explicitly stated otherwise. It is sufficient to note that, when an elliptical bell-shaped mountain is considered, such that

$$
h(x, y)=\frac{h_{0}}{\left[1+(x / a)^{2}+(y / b)^{2}\right]^{3 / 2}}, \quad \hat{h}^{\prime}=\frac{1}{2 \pi} e^{-\rho},
$$


$G=1 . B$ and $C$ may be expressed in terms of complete elliptical integrals of the first and second kind, as shown by Phillips (1984), but there is no advantage in repeating his calculations here. It suffices to note that, for an axisymmetric mountain, $B=C=\pi / 4$ and for a $2 \mathrm{D}$ mountain ridge, $B=1$ and $C=0$ (if the ridge axis is in the $y$ direction).

As in Teixeira et al. (2004) and Teixeira and Miranda (2004), the firstorder drag is zero, since the Fourier transform of the corresponding pressure perturbation (12) is real, being in phase (or out of phase by $\pi$ ) with the surface elevation. Hence

$$
D_{1 x}=D_{1 y}=0 .
$$

This explains why previous studies that calculated the drag using the 'standard' WKB solution (e.g. Shutts and Gadian 1999) failed to find any difference between the surface drag when the wind varies with height and when it is constant. In this standard solution, the series expansion of the vertical wavenumber (and thus the corresponding drag expansion) is considered only up to first order in $\varepsilon$, failing to capture the effects of shear and curvature of the wind profile.

It is only the second-order part of the drag that takes these effects into account:

$$
\begin{aligned}
D_{2 x}= & -\frac{1}{8} \rho_{0} N U_{0} b h_{0}^{2} G\left[I_{1}(\gamma)\left(\frac{\dot{U}_{0}^{2}}{N^{2}}+2 \frac{U_{0} \ddot{U}_{0}}{N^{2}}\right)\right. \\
& \left.+I_{2}(\gamma)\left(\frac{\dot{V}_{0}^{2}}{N^{2}}+2 \frac{V_{0}}{U_{0}} \frac{\dot{U}_{0} \dot{V}_{0}}{N^{2}}+2 \frac{V_{0}}{U_{0}} \frac{V_{0} \ddot{U}_{0}}{N^{2}}+4 \frac{V_{0} \ddot{V}_{0}}{N^{2}}\right)\right], \\
D_{2 y}= & -\frac{1}{8} \rho_{0} N V_{0} b h_{0}^{2} G \gamma\left[I_{1}(1 / \gamma)\left(\frac{\dot{V}_{0}^{2}}{N^{2}}+2 \frac{V_{0} \ddot{V}_{0}}{N^{2}}\right)\right. \\
& \left.+I_{2}(1 / \gamma)\left(\frac{\dot{U}_{0}^{2}}{N^{2}}+2 \frac{U_{0}}{V_{0}} \frac{\dot{U}_{0} \dot{V}_{0}}{N^{2}}+2 \frac{U_{0}}{V_{0}} \frac{U_{0} \ddot{V}_{0}}{N^{2}}+4 \frac{U_{0} \ddot{U}_{0}}{N^{2}}\right)\right],
\end{aligned}
$$

where

$$
\begin{aligned}
& I_{1}(\gamma)=\int_{0}^{\pi / 2} \frac{\cos ^{4} \theta}{\left(\cos ^{2} \theta+\gamma^{2} \sin ^{2} \theta\right)^{3 / 2}} d \theta \\
& I_{2}(\gamma)=\gamma^{2} \int_{0}^{\pi / 2} \frac{\cos ^{2} \theta \sin ^{2} \theta}{\left(\cos ^{2} \theta+\gamma^{2} \sin ^{2} \theta\right)^{3 / 2}} .
\end{aligned}
$$

It is possible to express $I_{1}$ and $I_{2}$ in terms of complete elliptical integrals of the first and second kind, but it is more useful to present them here instead in terms of $B$ and $C$, introduced above:

$$
\begin{aligned}
I_{1}(\gamma) & =\frac{B(\gamma)-C(\gamma)}{1-\gamma^{2}}, \quad I_{2}(\gamma)=\frac{C(\gamma)-\gamma^{2} B(\gamma)}{1-\gamma^{2}} \\
I_{1}(1 / \gamma) & =\frac{\gamma}{1-\gamma^{2}}(B(\gamma)-C(\gamma)), \quad I_{2}(1 / \gamma)=\frac{1 / \gamma}{1-\gamma^{2}}\left(C(\gamma)-\gamma^{2} B(\gamma)\right) .
\end{aligned}
$$

The latter two definitions were obtained from the first two by noting that $C(1 / \gamma)=(1 / \gamma) B(\gamma)$ and $B(1 / \gamma)=(1 / \gamma) C(\gamma)$ (Phillips 1984). 

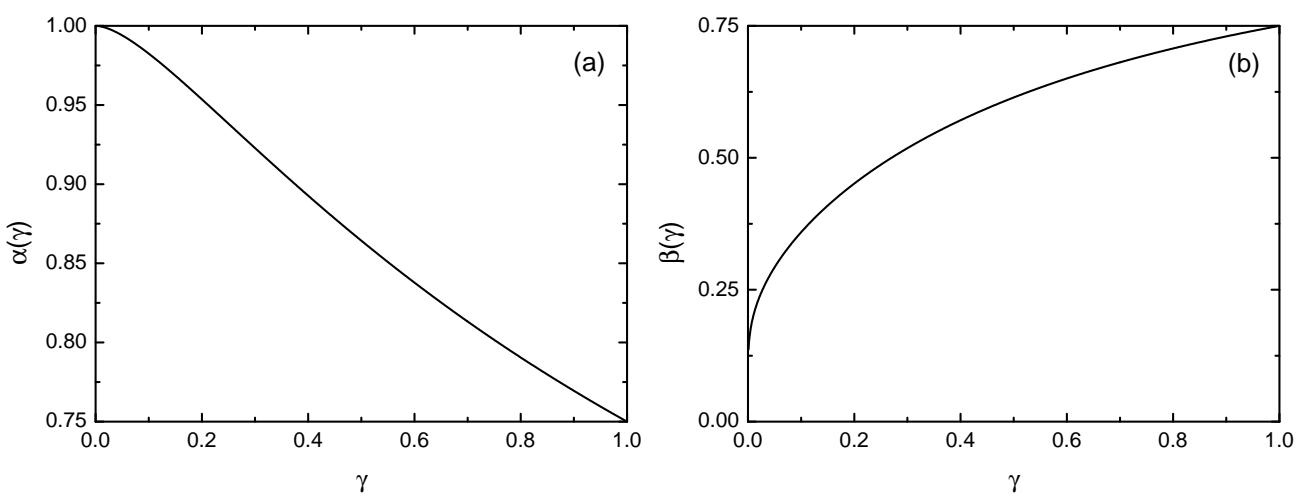

Figure 1. (a) Variation of $\alpha(\gamma)$ and of $\beta(1 / \gamma)$ for $0<\gamma<1$. (b) Variation of $\beta(\gamma)$ and of $\alpha(1 / \gamma)$ in the same interval.

In order to obtain the total drag (accurate to second order in $\varepsilon$ ), (19), (24) and (25)-(26) must be added. Since Phillips (1984) studied in detail the drag for a constant wind (here the zeroth-order term), it is perhaps more useful to build on his work by presenting the drag affected by shear and curvature of the wind profile as a correction to (19) (as was done by Teixeira et al. 2004 and Teixeira and Miranda 2004). This yields particularly simple expressions:

$$
\begin{aligned}
\frac{D_{x}}{D_{0 x}}= & 1-\frac{1}{8}\left[\alpha\left(\frac{U_{0}^{\prime 2}}{N^{2}}+2 \frac{U_{0} U_{0}^{\prime \prime}}{N^{2}}\right)\right. \\
& \left.+(1-\alpha)\left(\frac{V_{0}^{\prime 2}}{N^{2}}+2 \frac{V_{0}}{U_{0}} \frac{U_{0}^{\prime} V_{0}^{\prime}}{N^{2}}+2 \frac{V_{0}}{U_{0}} \frac{V_{0} U_{0}^{\prime \prime}}{N^{2}}+4 \frac{V_{0} V_{0}^{\prime \prime}}{N^{2}}\right)\right], \\
\frac{D_{y}}{D_{0 y}}= & 1-\frac{1}{8}\left[\beta\left(\frac{V_{0}^{\prime 2}}{N^{2}}+2 \frac{V_{0} V_{0}^{\prime \prime}}{N^{2}}\right)\right. \\
& \left.+(1-\beta)\left(\frac{U_{0}^{\prime 2}}{N^{2}}+2 \frac{U_{0}}{V_{0}} \frac{U_{0}^{\prime} V_{0}^{\prime}}{N^{2}}+2 \frac{U_{0}}{V_{0}} \frac{U_{0} V_{0}^{\prime \prime}}{N^{2}}+4 \frac{U_{0} U_{0}^{\prime \prime}}{N^{2}}\right)\right],
\end{aligned}
$$

where

$$
\begin{aligned}
& \alpha(\gamma)=\frac{I_{1}(\gamma)}{B(\gamma)}=1-\frac{I_{2}(\gamma)}{B(\gamma)}=\frac{1}{1-\gamma^{2}}\left(1-\frac{C(\gamma)}{B(\gamma)}\right) \\
& \beta(\gamma)=\frac{\gamma I_{1}(1 / \gamma)}{C(\gamma)}=1-\frac{\gamma I_{2}(1 / \gamma)}{C(\gamma)}=\frac{\gamma^{2}}{1-\gamma^{2}}\left(\frac{B(\gamma)}{C(\gamma)}-1\right) .
\end{aligned}
$$

There are several remarkable aspects in (31)-(32). First, the normalised drag expressions do not depend on the value of the integral $G$. So the results presented here are valid for any mountain that has an elliptical horizontal cross section. As suggested by the studies of Teixeira et al. (2004) and Teixeira and Miranda (2004), this appears to be a general property of hydrostatic flow, which presumably holds whenever the variation of the terrain elevation in the horizontal can be separated into a radial and an axial dependence.

Additionally, the coefficients $\alpha$ and $\beta$ only depend on the parameter $\gamma$, which quantifies the elongation of the elliptical orography (in Teixeira et al. 2004 and Teixeira and Miranda 2004, these coefficients were constant). Going back to 
the integral representations of $I_{1}, I_{2}, B$ and $C((21)-(22)$ and (27)-(28)), it can be shown that, for an axisymmetric mountain $(\gamma=1), \alpha=\beta=3 / 4$. With these values, (31)-(32) reproduce exactly the drag expressions of Teixeira et al (2004) (their equations (50)-(51)). For a $2 \mathrm{D}$ mountain ridge aligned in the $y$ direction, on the other hand, $\gamma=0$ and $\alpha=1$, so (31)-(32) reduce to equation (13) of Teixeira and Miranda (2004). For intermediate values of $\gamma$ (elliptical mountains), $\alpha$ and $\beta$ take intermediate values, as will be shown next. It may also be verified that $\beta(1 / \gamma)=\alpha(\gamma)$, which is a consequence of symmetry constraints.

Finally, it is noteworthy that, in (31)-(32), the normalised drag only depends on the values of the wind velocity and its vertical derivatives at the surface. This aspect, which is a consequence of the WKB approximation, means that, in a practical implementation of these formulae, the height where these quantities are evaluated has to be decided as objectively as possible. A leading-order guess would be to take the value of the velocity derivatives at the height where the velocity is evaluated in current GWD parameterisations, but more refined procedures could be developed.

In Fig. 1, $\alpha$ and $\beta$ are plotted as functions of $\gamma$. In Fig. 1(a), $\alpha$ is shown for $0<\gamma<1$ and, for the reasons presented above, the curve also describes the variation of $\beta$ between $\infty$ and 1. In Fig. 1(b), $\beta$ is shown for $0<\gamma<1$, which also corresponds to $\alpha$ between $\infty$ and 1. Figure 1(a) shows that $\alpha$ decreases from 1 to 0.75 as $\gamma$ goes from 0 to 1 . This is the reason why corrections due to shear and curvature become more important as one shifts from an axisymmetric to a 2D mountain perpendicular to the wind (cf. Teixeira et al. 2004, Teixeira and Miranda 2004). In Fig. 1(b) it can be seen that, as $\gamma$ goes from 0 to $1, \beta$ goes from 0 to 0.75 , but rises extremely rapidly near $\gamma=0$. This means that the impact of shear on the drag in the direction of the longest axis of an elliptical mountain persists even when the mountain is very elongated.

\section{(a) A few examples}

Flow over a ridge and flow over an axisymmetric mountain can be considered limit cases of flow over an elliptical mountain. As seen above, the behaviour of $\alpha$ and $\beta$ is such that the normalised drag is affected by the wind profile to an intermediate degree between those two limits. Hence no surprising results, relative to those predicted in the studies of Teixeira et al. (2004) and Teixeira and Miranda (2004) would be expected. However, the following examples, for a wind that varies linearly or turns with height at a constant rate, contradict this idea. In polar coordinates, it is particularly clear that these are the simplest possible wind profiles with a constant Richardson number, $R i=N^{2} /\left(U^{\prime 2}+V^{\prime 2}\right)$, since one has a constant azimuthal angle and a linearly varying wind speed, while the other has a constant wind speed and a linearly varying azimuthal angle. Following Phillips (1984), an elliptical mountain of aspect ratio $\gamma=0.5$ (therefore with the major axis aligned in the $y$ direction) will be considered.

(i) Backward linear wind profile

A backward linear wind profile is assumed here. As pointed out in Teixeira et al. (2004), this profile is especially suitable for testing the dependence of the drag on the Richardson number, because the wave energy is absorbed at the critical level for moderate $R i$, at least for relatively low mountains. This precludes spurious wave reflections, which would complicate the dynamics of the processes determining the drag (Teixeira et al. 2005, Teixeira and Miranda 2005). 
Figure 2 shows the normalised drag given by (31)-(32) (straight lines) against data from simulations of a mesoscale non-hydrostatic nonlinear numerical model (NH3D) (symbols). The numerical model (described in Miranda and James 1992) considered approximately linear and hydrostatic conditions $\left(\mathrm{Na} / U_{0}=45.26\right.$ and $\left.N h_{0} / U_{0}=0.01\right)$ in order to focus only on wind profile effects. It used a grid of $65 \times 65 \times 300$ points, with a horizontal spacing of $4 \mathrm{~km}$ and a vertical spacing varying between 22 and $72 \mathrm{~m}$ (with the highest resolution near the ground) and was run for a time sufficient for the drag to attain a steady state. The lower boundary condition was free-slip and the lateral and upper boundary conditions used sponges, where the flow was relaxed toward its background value, with relaxation times of 50 and 20 timesteps, respectively. The height of the lower limit of the upper sponge was $8 \mathrm{~km}$, which is above the critical level in all the sheared flows considered.

Also shown (solid and dashed lines) are curves corresponding to exact linear theory (valid for arbitrary shear rates), which can be considered an extension of the theory developed by Grubišić and Smolarkiewicz (1997) for axisymmetric mountains. The GWD is obtained from this exact theory by following the same analytical procedure as described previously for the WKB model, but using instead the exact solution for $\hat{w}$ that can be determined when both $U^{\prime}$ and $V^{\prime}$ are constant:

$$
\hat{w}=\hat{w}(z=0)\left(1+\frac{U^{\prime} k_{1}+V^{\prime} k_{2}}{U_{0} k_{1}+V_{0} k_{2}} z\right)^{\frac{1}{2}+i \mu \operatorname{sign}\left(U^{\prime} k_{1}+V^{\prime} k_{2}\right)},
$$

where

$$
\mu=\sqrt{\frac{N^{2} k_{12}^{2}}{\left(U^{\prime} k_{1}+V^{\prime} k_{2}\right)^{2}}-\frac{1}{4}} .
$$

In Fig. 2(a), results for a wind perpendicular to the major axis of the mountain (along $x$ ) and a wind parallel to the major axis (along $y$ ) are shown. The corresponding wind profiles are, respectively:

$$
\begin{aligned}
& U=U_{0}-c_{1} z, \quad V=0, \\
& U=0, \quad V=V_{0}-c_{2} z,
\end{aligned}
$$

where $U_{0}, V_{0}, c_{1}, c_{2}>0$ are constants. The normalised drag given by (31)-(32) for these cases is, respectively:

$$
\begin{aligned}
& \frac{D_{x}}{D_{0 x}}=1-\frac{\alpha}{8 R i}, \\
& \frac{D_{y}}{D_{0 y}}=1-\frac{\beta}{8 R i} .
\end{aligned}
$$

(The components of the drag transverse to the wind are zero by symmetry).

The exact linear drag is given by

$$
\begin{aligned}
& \frac{D_{x}}{D_{0 x}}=\frac{1}{4 B(\gamma)} \int_{0}^{2 \pi} \frac{\cos ^{2} \theta}{\left(\cos ^{2} \theta+\gamma^{2} \sin ^{2} \theta\right)^{1 / 2}}\left[1-\frac{1}{4 R i} \frac{\cos ^{2} \theta}{\cos ^{2} \theta+\gamma^{2} \sin ^{2} \theta}\right]^{1 / 2} d \theta, \\
& \frac{D_{y}}{D_{0 y}}=\frac{\gamma^{2}}{4 C(\gamma)} \int_{0}^{2 \pi} \frac{\sin ^{2} \theta}{\left(\cos ^{2} \theta+\gamma^{2} \sin ^{2} \theta\right)^{1 / 2}}\left[1-\frac{1}{4 R i} \frac{\gamma^{2} \sin ^{2} \theta}{\cos ^{2} \theta+\gamma^{2} \sin ^{2} \theta}\right]^{1 / 2} d \theta,
\end{aligned}
$$



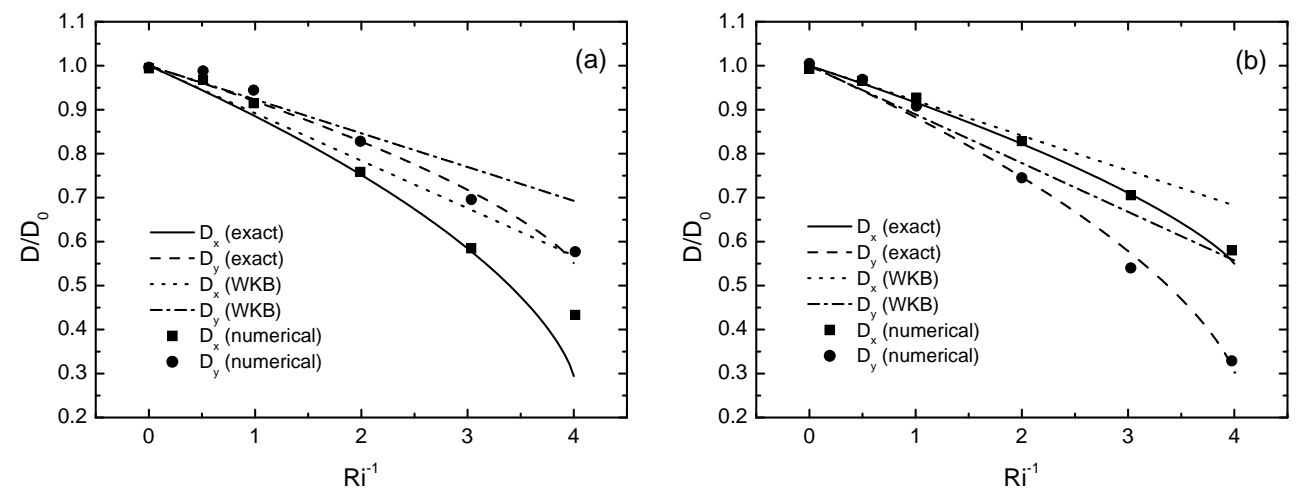

Figure 2. Normalised drag as a function of $R i^{-1}$. Solid line: $D_{x} / D_{0 x}$ from exact linear theory, dashed line: $D_{y} / D_{0 y}$ from exact linear theory, dotted line: $D_{x} / D_{0 x}$ from WKB theory, dash-dotted line: $D_{y} / D_{0 y}$ from WKB theory, squares: $D_{x} / D_{0 x}$ from numerical simulations, circles: $D_{y} / D_{0 y}$ from numerical simulations. (a) Wind profiles (37) (for $D_{x} / D_{0 x}$ ) and (38) (for $D_{y} / D_{0 y}$ ) (b) Wind profile (43) (for both $D_{x} / D_{0 x}$ and $\left.D_{y} / D_{0 y}\right)$.

respectively. These equations are analogous to eq. (28) of Grubišić and Smolarkiewicz (1997), but consider a mountain with an elliptical cross-section. It can be shown, by Taylor-expanding (41)-(42) for large $R i$, that these formulae tend asymptotically to (39)-(40).

In Fig. 2(b), the drag for a wind at a 45 degree angle to the main axes of the mountain (bisecting the first quadrant) is shown. It corresponds to the following wind profile:

$$
U=U_{0}-c_{1} z, \quad V=V_{0}-c_{2} z,
$$

where $U_{0}=V_{0}>0$ and $c_{1}=c_{2}>0$ are constants. In that case (31)-(32) reduce to

$$
\frac{D_{x}}{D_{0 x}}=1-\frac{1}{16 R i}(3-2 \alpha), \quad \frac{D_{y}}{D_{0 y}}=1-\frac{1}{16 R i}(3-2 \beta),
$$

and the exact linear drag may be shown to be given by

$$
\begin{aligned}
& \frac{D_{x}}{D_{0 x}}=\frac{1}{4 B(\gamma)} \int_{0}^{2 \pi} \frac{\cos \theta(\cos \theta+\gamma \sin \theta)}{\left(\cos ^{2} \theta+\gamma^{2} \sin ^{2} \theta\right)^{1 / 2}}\left[1-\frac{1}{8 R i} \frac{(\cos \theta+\gamma \sin \theta)^{2}}{\cos ^{2} \theta+\gamma^{2} \sin ^{2} \theta}\right]^{1 / 2} d \theta, \\
& \frac{D_{y}}{D_{0 y}}=\frac{\gamma}{4 C(\gamma)} \int_{0}^{2 \pi} \frac{\sin \theta(\cos \theta+\gamma \sin \theta)}{\left(\cos ^{2} \theta+\gamma^{2} \sin ^{2} \theta\right)^{1 / 2}}\left[1-\frac{1}{8 R i} \frac{(\cos \theta+\gamma \sin \theta)^{2}}{\cos ^{2} \theta+\gamma^{2} \sin ^{2} \theta}\right]^{1 / 2} d \theta .
\end{aligned}
$$

These equations also tend asymptotically to (44) for high $R i$.

As can be seen, in Fig. 2 the agreement of the numerical data with the exact analytical theory is quite good. The agreement of the WKB theory (44) with the numerical data is less good, especially for low $R i$, as would be expected, but the trends displayed are correct.

It should be pointed out that, since for $\gamma=0.5, \alpha=0.864$ and $\beta=0.614$, according to (39)-(40), the drag in a flow perpendicular to the major axis of the mountain is more affected by shear than the drag in a flow parallel to that axis. But in a flow at a 45 degree angle to the axes of the mountain, the component of 

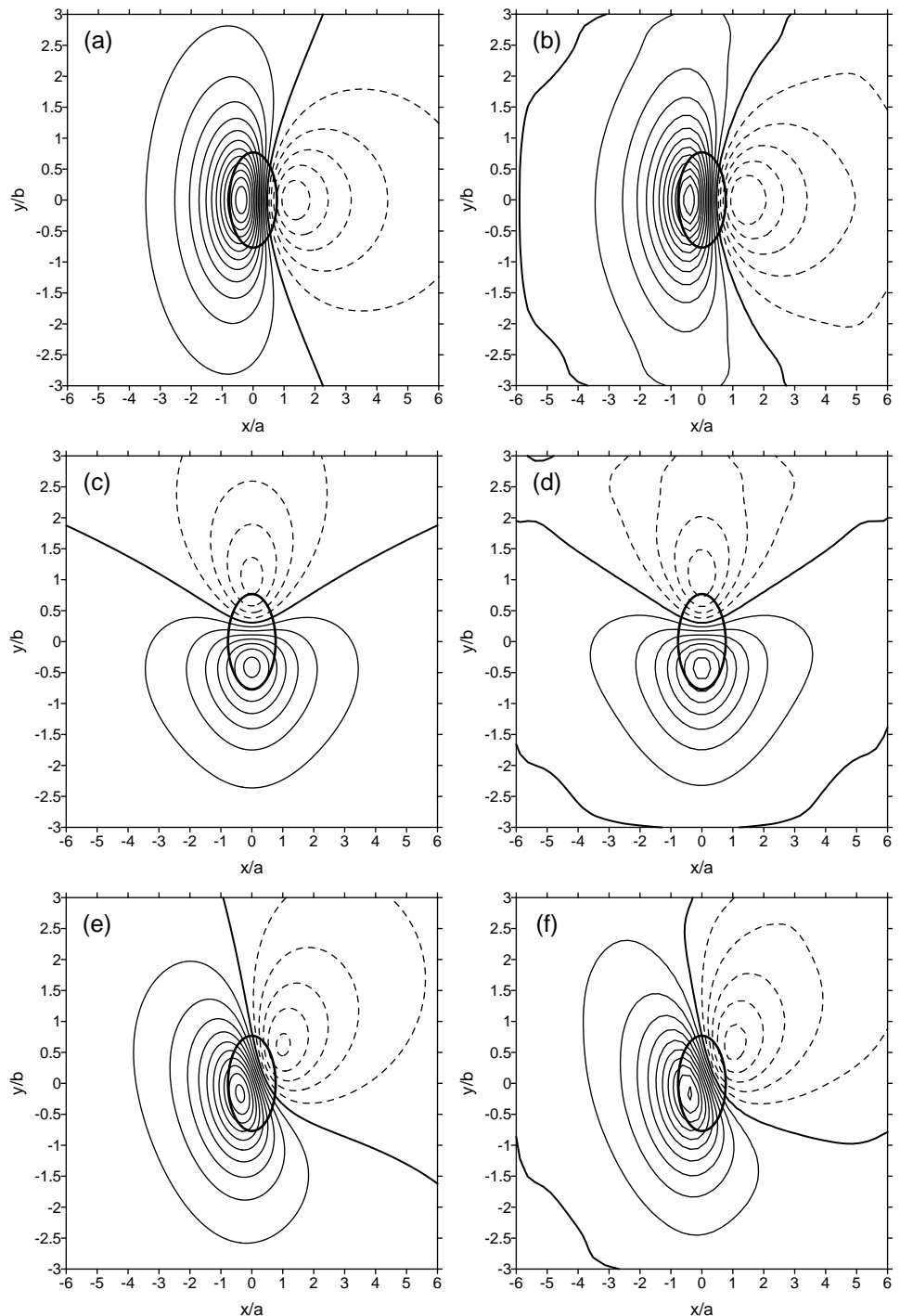

Figure 3. Normalised pressure perturbation, $p /\left(\rho_{0} N\left(U_{0}^{2}+V_{0}^{2}\right)^{1 / 2} h_{0}\right)$ for $R i=0.5$. Solid lines: positive values, dashed lines: negative values. Thick solid line: $h(x, y) / h_{0}=0.5$ Contour spacing: 0.05 . (a), (c) and (e): WKB theory. (b), (d) and (f): numerical simulations. (a) and (b): wind profile (37), (c) and (d): wind profile (38), (e) and (f): wind profile (43).

the drag most affected by the shear is not the $x$ component but the $y$ component, due to the peculiar dependence on $\alpha$ and $\beta$ existing in that case (see (44)). Note that, between (39)-(40) and (44), the dependence of the drag on $\alpha$ and $\beta$ has opposite signs. This behaviour is caused by the existence of terms involving the products of $U_{0}^{\prime}$ and $V_{0}^{\prime}$ in (31)-(32). Nevertheless, both (39)-(40) and (44) reduce to eq. (53) of Teixeira et al. (2004) for an axisymmetric mountain $(\alpha=\beta=0.75)$.

Figure 3 shows the surface pressure perturbations corresponding to the flows (37), (38) and (43), at $R i=0.5$, for a mountain given by (23), resulting from the WKB model developed here and from simulations of the NH3D numerical model, using the same flow parameters as in Fig. 2. As can be seen, both the 
magnitude and the structure of the pressure perturbation are well reproduced, except towards the outer edges of the domain, where the effect of the boundary conditions appears to slightly affect the numerical simulations.

For an elliptical mountain of the same horizontal aspect ratio (albeit using an exponent of 2 instead of 1.5 in the denominator of (23)), Phillips (1984) determined the pressure perturbation analogous to those presented in Fig. 3. Since he did not include shear in his analytical model, the pressure field shown in his Fig. 2 is anti-symmetric, although displaying different types of elongation and shape depending on whether the flow is perpendicular, parallel or at a 45 degree angle to the main axes of the mountain. In this respect, rotating his panels so that the major axis of the mountain becomes vertical, his graphs may be qualitatively compared with the present ones. There is some similarity in the distortion of the pressure perturbation, dictated by the approximately similar shape of the mountain. However, due to shear, the pressure pattern in Fig. 3 is not antisymmetric but wedge-shaped, with the pressure maximum translated toward the mountain top (as happened in Teixeira et al. (2004) for an axisymmetric mountain but the same type of flow).

Since the downstream displacement of the pressure perturbation is determined by the wind profile, but the distance required for this displacement to decrease the drag is smaller when the wind is perpendicular to the major axis of the mountain (Fig. 3(a),(b)) than when it is parallel (Fig. 3(c),(d)), this explains why the drag is more affected by shear in the former case than in the latter in Fig. 2(a).

\section{(ii) Wind that turns with height}

As in Teixeira et al. (2004), a wind that rotates with height at a constant rate, albeit retaining its magnitude, is now considered. Like the one considered in the previous section, this wind profile is characterised by a constant $R i$, and it also leads to absorption of upward-propagating waves, precluding reflections. However, no exact analytical theory exists for this case, therefore a linear, but numerical, model is employed in conjunction with the WKB theory and the fully nonlinear numerical model. The linear model is described in Appendix A.

Generically, such a wind profile is given by

$$
U=U_{0} \cos \left(c_{3} z+c_{4}\right), \quad V=U_{0} \sin \left(c_{3} z+c_{4}\right),
$$

where $c_{3}$ and $c_{4}$ are constants. Here it will be assumed that $c_{3}>0$, corresponding to an anti-clockwise rotation of the wind as $z$ increases.

As in the previous section, an elliptical mountain of aspect ratio $\gamma=0.5$ is considered, and three situations are addressed: a wind along $x$ at the surface $\left(c_{4}=0\right)$, a wind along $y$ at the surface $\left(c_{4}=\pi / 2\right)$, and a wind whose direction bisects the first quadrant at the surface $\left(c_{4}=\pi / 4\right)$. In Fig $4(\mathrm{a}), D_{x} / D_{0 x}$ is presented as a function of $R i$ for the first case and $D_{y} / D_{0 y}$ for the second. In Fig. 4(b), both $D_{x} / D_{0 x}$ and $D_{y} / D_{0 y}$ are presented for the third case. The symbols correspond to simulations of the NH3D numerical model, the solid and dashed lines to the predictions of the linear numerical model presented in Appendix A, and the dotted and dash-dotted lines to the predictions of the WKB model. The numerical parameters used in the NH3D model are the same as for Fig. 2.

For the cases of Fig. 4(a), the WKB model predicts that there is no component of the drag perpendicular to the wind at the surface, and that the normalised drag along $x$ in the first case and the normalised drag along $y$ in the 

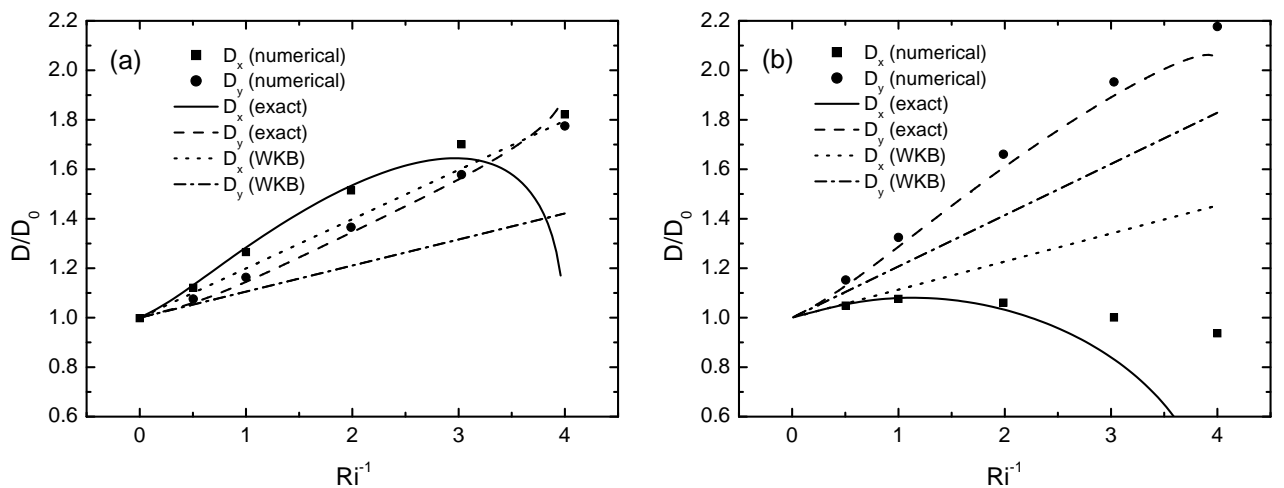

Figure 4. Normalised drag as a function of $R i^{-1}$ for the wind profile (47). Solid line: $D_{x} / D_{0 x}$ from linear numerical model, dashed line: $D_{y} / D_{0 y}$ from linear numerical model, dotted line: $D_{x} / D_{0 x}$ from WKB theory, dash-dotted line: $D_{y} / D_{0 y}$ from WKB theory, squares: $D_{x} / D_{0 x}$ from numerical simulations, circles: $D_{y} / D_{0 y}$ from numerical simulations. (a) $c_{4}=0$ (for $D_{x} / D_{o x}$ ) and $c_{4}=\pi / 2$ (for $D_{y} / D_{0 y}$ ), (b) $c_{4}=\pi / 4\left(\right.$ for both $D_{x} / D_{0 x}$ and $\left.D_{y} / D_{0 y}\right)$.

second are given, respectively, by

$$
\begin{aligned}
& \frac{D_{x}}{D_{0 x}}=1+\frac{1}{8 R i}(3 \alpha-1), \\
& \frac{D_{y}}{D_{0 y}}=1+\frac{1}{8 R i}(3 \beta-1) .
\end{aligned}
$$

In the case presented in Fig. 4(b), the two components of the drag are predicted to be

$$
\frac{D_{x}}{D_{0 x}}=1+\frac{1}{16 R i}(7-6 \alpha), \quad \frac{D_{y}}{D_{0 y}}=1+\frac{1}{16 R i}(7-6 \beta) .
$$

It should be noted that, for a circular mountain $(\alpha=\beta=3 / 4)$, both (48)(49) and (50) reduce to eq. (57) of Teixeira et al. (2004). Additionally, as for a linear wind profile, the dependence of the drag components on $\alpha$ and $\beta$ is in opposite senses between (48)-(49) and (50). This implies that, although the drag generally increases as $R i$ decreases, the drag for a flow along the major axis of the mountain has a considerably weaker dependence on $R i$ than the drag in flow across the major axis of the mountain. However, for a wind at a 45 degree angle to the main axes of the mountain, the drag component along $x$ is considerably less dependent on $R i$ than the drag component along $y$. This result is, once again, due to the existence of products between $U_{0}^{\prime}$ and $V_{0}^{\prime}$ in the original drag expressions, (31)-(32).

There is an important difference: while in (39), (40) and (44), the coefficient multiplying $R i^{-1}$ never changes sign (albeit being of variable magnitude), since $\alpha$ and $\beta$ vary between 0 and 1 , in (48) or (49), this coefficient may change sign. This means that the normalised drag may either increase or decrease as $R i$ decreases, depending on the elongation of the mountain. In practise, the drag is almost always predicted to increase as $R i$ decreases, and the elongation of the mountain in the surface wind direction must be very large, of order $1 / 36$, for the drag to start decreasing as $R i$ decreases.

In Fig. 4 it can be seen that results predicted by the WKB tend asymptotically to those of the linear numerical model for high $R i$, as required. However, 

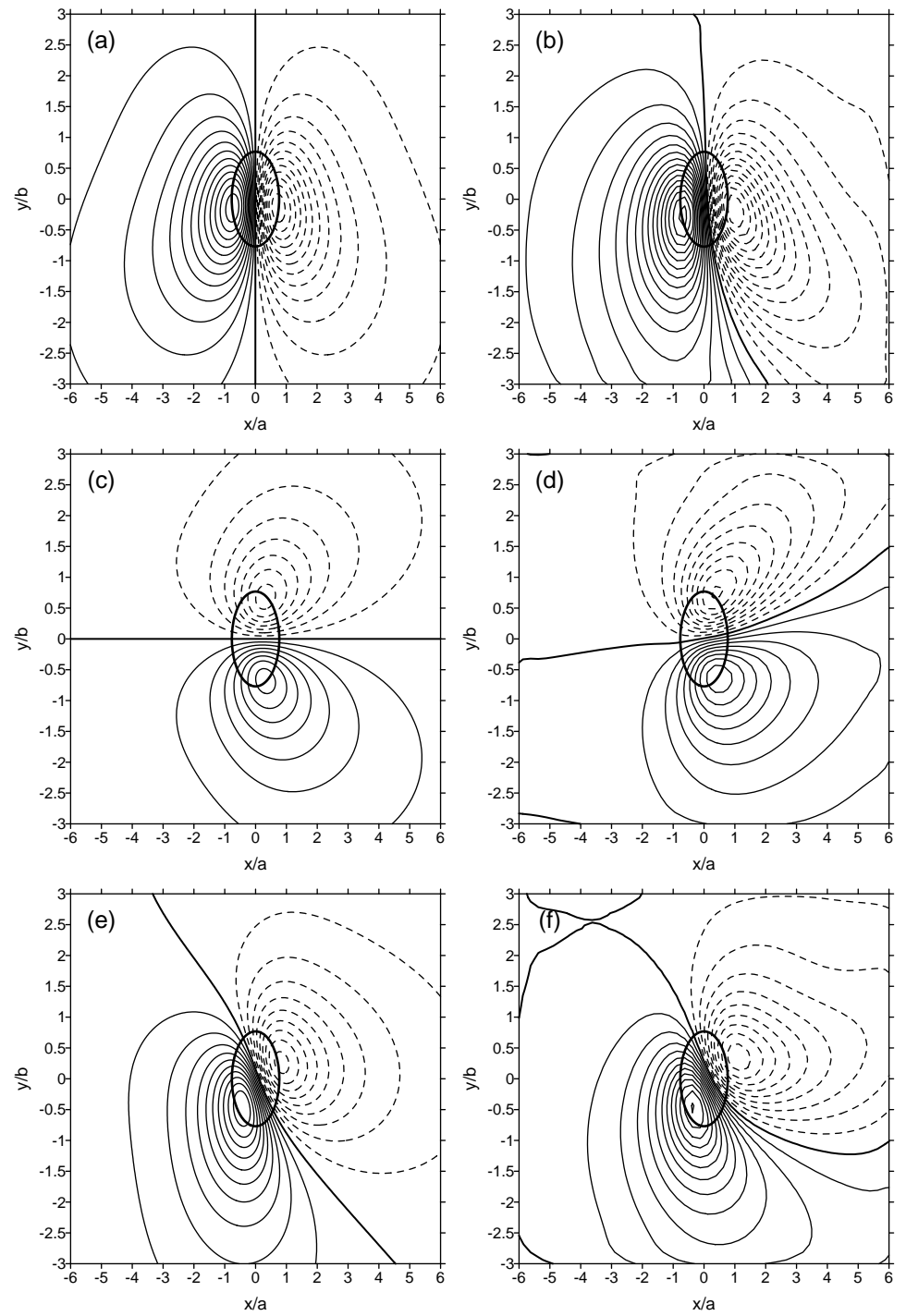

Figure 5. Normalised pressure perturbation, $p /\left(\rho_{0} N\left(U_{0}^{2}+V_{0}^{2}\right)^{1 / 2} h_{0}\right)$ for $R i=0.5$ for the wind profile (47). Solid lines: positive values, dashed lines: negative values. Thick solid line: $h(x, y) / h_{0}=0.5$. Contour spacing: 0.05. (a), (c) and (e): WKB theory. (b), (d) and (f): numerical simulations. (a) and (b): $c_{4}=0$, (c) and (d): $c_{4}=\pi / 2$, (e) and (f): $c_{4}=\pi / 4$.

departures at lower $R i$ are considerable, undoubtedly more important than those observed for a linear wind profile. In Fig 4(a), for example, $D_{x} / D_{0 x}$ starts by increasing faster than predicted by the WKB model as $R i$ decreases, but then, at about $R i^{-1}=3$, begins decreasing. It has been verified that this effect only occurs for flow across the mountain. For flow along the mountain, $D_{y} / D_{0 y}$ always increases faster than predicted by the WKB model. Figure 4(b) shows that departures of the WKB model from the linear numerical model are even larger for oblique flow. In this case $D_{x} / D_{0 x}$ changes its dependence from increasing to decreasing as $R i$ decreases at about $R i=1$, and $D_{y} / D_{0 y}$ is always above the WKB result. This behaviour may be attributed in part to the fact that the drag 
modulus starts decreasing for sufficiently low $R i$ (as in Fig 4(a)) and partly to the fact that the drag is not, in fact, aligned with the surface wind, as predicted by the WKB model, but rotates to the left, like the wind. This effect, which is particularly marked at low $R i$, leads to some transfer of drag from the $x$ to the $y$ component.

In Fig. 4, the results from the NH3D numerical model are generally in good agreement with the linear exact results except for $R i=0.25$, where they are slightly closer to the WKB results. This could be a consequence of the smoothing effect of the finite vertical resolution of the NH3D model (due to its method of solution, the linear numerical model has arbitrarily high vertical resolution). Additional numerical simulations (not shown) suggest that doubling the vertical resolution of the NH3D model brings the values of the drag at low $R i$ nearer to those predicted by the linear numerical model, but the agreement is not perfect. There is a possibility that nonlinear effects play a role in the observed differences, even for the extremely low mountains considered.

Figure 5 shows the pressure perturbation at the surface for $R i=0.5$, for the wind profile (47) at various incidence angles, from the WKB model and from NH3D, for a mountain given by (23). The parameters used in NH3D are the same considered in the results of Fig. 4. The pressure perturbation is considerably more intense than in Fig. 3, which is consistent with the generally higher drag. It has a shape resembling that calculated by Teixeira et al. (2004) for a circular mountain, with maxima and minima possessing lobes elongated to the right of the surface wind. But there is additional distortion introduced by the elliptical orography. The agreement with the numerical results is also worse than in Fig. 3, which is consistent with the worse prediction of the drag. The pressure perturbation is generally more intense in the numerical results, as indicated by the larger number of contours in Figs. 5(b),(d),(f), and also rotated to the left relative to the results from the WKB model, presented in Figs. 5(a),(c),(e).

From the viewpoint of internal gravity wave structure, the fact that a linearly decreasing wind leads to a decrease of the drag while a turning wind leads to an increase the drag as $R i$ decreases is due to the distinct effects that shear and curvature of the wind profile have on the vertical wavenumber of the gravity waves. It can be shown that GWD is essentially proportional to the real part of the vertical wavenumber of the waves (see (A.10) of Appendix A), and while shear (of any sign) leads to a decrease of this quantity, negative curvature (such as exists in a turning wind) leads instead to an increase. This explains why the second-order part of the wave solution (13) produces a pressure perturbation that weakens the zeroth-order part in the first case, while it reinforces the zeroth-order part in the second. These aspects are discussed in detail in Teixeira et al. (2004).

Up to now, a frame of reference that is aligned with the main axes of the elliptical mountain has been used, because the terrain elevation is expressed most easily in that case. For the purpose of parameterising the GWD, it would perhaps be more convenient to adopt polar coordinates, since these have no preferential direction, and the drag expressions can be cast in a form that only depends on the wind magnitude and the angle between the wind and the axes of the orography. Unfortunately, the drag expressions in polar coordinates are exceedingly lengthy (see Appendix B), and provide no additional insight into the underlying physics. Therefore, in drag calculations, it is probably more advantageous to use the original drag expressions (31)-(32) by adopting a coordinate system aligned with the main axes of the mountain. 


\section{CONCLUDING REMARKS}

In this study, an analytical model of gravity wave drag produced by mountains is developed. The model is inviscid, linear and hydrostatic, and considers generic wind profiles that vary relatively slowly in the vertical. The mountains considered have an elliptical horizontal cross-section. Subject to these conditions, the Taylor-Goldstein equation is solved using a 2nd order WKB approximation to obtain the internal gravity wave solutions. It is found that the drag exerted by the flow on the mountains normalised by its value for a constant wind equal to the surface wind depends, for the simplest flows, on only two parameters: the Richardson number at the surface, $R i$, and the aspect ratio of the mountain, $\gamma$. The model is tested for two simple wind profiles with constant $R i$, which serve to illustrate its range of behaviour.

For a linear wind profile, the normalised drag is found to decrease as $R i$ decreases, faster for a wind that is perpendicular to the major axis of the mountain than for a wind that is parallel to the major axis of the mountain. However, for a wind at a 45 degree angle to the main axes of the mountain, the component of the drag most affected by shear is that along the major axis of the mountain.

For a wind that turns with height maintaining its magnitude, the normalised drag generally increases as $R i$ decreases, with a faster variation when the wind is across the mountain than when the wind is along it, as in the preceding case. But for mountains that are very elongated in the direction of the surface wind, this dependence may change sign. In fact, for this type of flow, the predictions from the WKB model are seen to be less satisfactory than for a linear wind profile.

However, it should be noted that, in all cases, the WKB model developed here is asymptotically correct in the limit of high $R i$, an aspect that is confirmed by the numerical simulations. And the WKB model clearly provides an improvement on Phillips' (1984) drag model, which it originally aimed to extend.

For these reasons, and also because elliptical mountains are used in existing drag parametrisation schemes as building blocks to represent the real global orography, it is expected that the present calculations facilitate the implementation of wind profile corrections to those schemes.

\section{Appendix A}

\section{Linear numerical model}

The numerical model that is used in section 3(a)(ii) to calculate the exact linear drag (i.e. for arbitrarily large shears) for a wind that turns with height is described next (cf. Sivertsen 1972).

Without any loss of generality, the solution to the Taylor-Goldstein equation, (1), may be cast in the form

$$
\hat{w}=\hat{w}(z=0) \exp \left[i \int_{0}^{z} m(\zeta) d \zeta\right],
$$

which may be thought of as an extension of (4) (written in non-scaled coordinates) to the case where the vertical wavenumber $m$ is not expanded in a power series. In this expression, $\hat{w}(z=0)$ is provided by the lower boundary condition, (2).

When (A.1) is introduced into (1), the following equation results:

$$
i m^{\prime}-m^{2}+\frac{N^{2} k_{12}^{2}}{\left(U k_{1}+V k_{2}\right)^{2}}-\frac{U^{\prime \prime} k_{1}+V^{\prime \prime} k_{2}}{U k_{1}+V k_{2}}=0 .
$$


Despite being nonlinear, relative to the Taylor-Goldstein equation, this equation for $m$ has the advantage of being only first-order, requiring just one boundary condition. This boundary condition is applied at the top of the atmosphere, and is easier to implement than the usual radiation boundary condition. If the flow reaches a zone where both $N$ and $(U, V)$ are constant with height with values, say $N_{\infty}$ and $\left(U_{\infty}, V_{\infty}\right)$, respectively, the boundary condition states that

$$
m(z \rightarrow+\infty)=\frac{N_{\infty} k_{12}}{U_{\infty} k_{1}+V_{\infty} k_{2}} .
$$

If, on the other hand, there is a critical level at some height $z_{c}$, the boundary condition requires that $m\left(z_{c}\right)=\infty$.

In the flow under consideration (a wind that turns with height indefinitely), the relevant upper boundary condition is the latter one, but the presence of the infinity in its specification is numerically inconvenient. For this reason, it is better to derive an equation for the inverse of the vertical wavenumber, more exactly the wavelength divided by $2 \pi$, which is called here $L=1 / \mathrm{m}$. It can be shown from (A.2) that this equation takes the form

$$
L^{\prime}=i\left\{1-\left[\frac{N^{2} k_{12}^{2}}{\left(U k_{1}+V k_{2}\right)^{2}}-\frac{U^{\prime \prime} k_{1}+V^{\prime \prime} k_{2}}{U k_{1}+V k_{2}}\right] L^{2}\right\} .
$$

It is clear that there is a singularity in (A.4) at critical levels, since in that case both $U k_{1}+V k_{2}$ in the denominator of the fractions and $L$ tend to zero. This leads to numerical problems, which can be avoided by adopting near critical levels the so-called Frobenius solution (Grubišić and Smolarkiewicz 1997). In terms of $L$, this solution is expressed as

$$
L=\frac{z-z_{c}}{-\frac{i}{2} \pm\left(\frac{N^{2} k_{12}^{2}}{\left(U_{c}^{\prime} k_{1}+V_{c}^{\prime} k_{2}\right)^{2}}-\frac{1}{4}\right)^{1 / 2}},
$$

where $\left(U_{c}, V_{c}\right)=(U, V)\left(z=z_{c}\right)$ and the sign in the denominator is determined by the condition that the wave energy propagates upward.

Since $L=L_{R}+i L_{I}$ is in general complex, in practise (A.4) must be split into two equations, for its real and imaginary parts:

$$
\begin{aligned}
L_{R}^{\prime} & =2\left[\frac{N^{2} k_{12}^{2}}{\left(U k_{1}+V k_{2}\right)^{2}}-\frac{U^{\prime \prime} k_{1}+V^{\prime \prime} k_{2}}{U k_{1}+V k_{2}}\right] L_{R} L_{I}, \\
L_{I}^{\prime} & =1+\left[\frac{N^{2} k_{12}^{2}}{\left(U k_{1}+V k_{2}\right)^{2}}-\frac{U^{\prime \prime} k_{1}+V^{\prime \prime} k_{2}}{U k_{1}+V k_{2}}\right]\left(L_{I}^{2}-L_{R}^{2}\right) .
\end{aligned}
$$

The equations to use near critical levels result from (A.5), and are:

$$
\begin{aligned}
L_{R}^{\prime} & =\frac{U_{c}^{\prime} k_{1}+V_{c}^{\prime} k_{2}}{N k_{12}}\left(1-\frac{1}{4} \frac{\left(U_{c}^{\prime} k_{1}+V_{c}^{\prime} k_{2}\right)^{2}}{N^{2} k_{12}^{2}}\right)^{1 / 2}, \\
L_{I}^{\prime} & =\frac{1}{2} \frac{\left(U_{c}^{\prime} k_{1}+V_{c}^{\prime} k_{2}\right)^{2}}{N^{2} k_{12}^{2}},
\end{aligned}
$$

where (A.8) already corresponds to upward wave energy propagation. These equations are integrated numerically, for a range of wavenumbers, from the top of 
the domain to the surface, using a 4th order Runge-Kutta method with automatic step size reduction. The relative precision imposed is $4 \times 10^{-5}$.

The ultimate aim of this model is to calculate the surface drag, which in wavenumber space is given by

$$
\left(D_{x}, D_{y}\right)=4 \pi^{2} \rho_{0} \int_{-\infty}^{+\infty} \int_{-\infty}^{+\infty} \frac{\left(k_{1}, k_{2}\right)}{k_{12}^{2}}\left(U_{0} k_{1}+V_{0} k_{2}\right)^{2} \operatorname{Re}[m(z=0)]|\hat{h}|^{2} d k_{1} d k_{2} .
$$

The real part of the wavenumber at the surface is easily calculated from the $L(z=0)$ determined as a solution to (A.6)-(A.7), through

$$
\operatorname{Re}[m(z=0)]=\frac{L_{R}(z=0)}{L_{R}^{2}(z=0)+L_{I}^{2}(z=0)} .
$$

On the other hand, introducing the elliptical polar coordinates (18) in (A.10) and normalising these drag expressions by their value in the absence of wind shear, the integrals over the radial coordinate cancel, so only 1D integrals over the azimuthal directions have to be calculated. The final drag expressions are:

$$
\begin{aligned}
\frac{D_{x}}{D_{0 x}} & =\frac{\int_{0}^{2 \pi} \frac{\cos \theta}{\cos ^{2} \theta+\gamma^{2} \sin \theta}\left(U_{0} \cos \theta+\gamma V_{0} \sin \theta\right)^{2} \operatorname{Re}[m(z=0)] d \theta}{N \int_{0}^{2 \pi} \frac{\cos \theta}{\left(\cos ^{2} \theta+\gamma^{2} \sin ^{2} \theta\right)^{1 / 2}}\left(U_{0} \cos \theta+\gamma V_{0} \sin \theta\right) d \theta}, \\
\frac{D_{y}}{D_{0 y}} & =\frac{\int_{0}^{2 \pi} \frac{\gamma \sin \theta}{\cos ^{2} \theta+\gamma^{2} \sin \theta}\left(U_{0} \cos \theta+\gamma V_{0} \sin \theta\right)^{2} \operatorname{Re}[m(z=0)] d \theta}{N \int_{0}^{2 \pi} \frac{\gamma \sin \theta}{\left(\cos ^{2} \theta+\gamma^{2} \sin ^{2} \theta\right)^{1 / 2}}\left(U_{0} \cos \theta+\gamma V_{0} \sin \theta\right) d \theta} .
\end{aligned}
$$

In (A.12)-(A.13), the fact that $\operatorname{Re}[m(z=0)]$ only depends on the azimuthal angle, which can be deduced from (A.2) and (A.3), was taken into account.

The solution procedure is the following: using a Gauss-Legendre integration method, a set of azimuthal angles for the vertical wavenumber (or the vertical wavelength) is selected. Then, (A.4) is solved for these azimuthal angles. Finally, the values of $\operatorname{Re}[m(z=0)]$ obtained from (A.11) for these azimuthal angles are introduced into (A.12)-(A.13), and the integrals present in these expressions are calculated numerically. In the calculations presented, the number of integration points is 200 .

This method of solution of the vertical structure equation for the internal gravity waves is alternative to that employed by Vosper (1995), being especially suitable for the calculation of the surface drag.

\section{APPENDIX B}

\section{Expressions of the GWD in polar coordinates}

The drag expressions (31)-(32) can be cast into a form that only depends on the wind magnitude and the angle between the wind and the axes of the orography, $\psi$. In polar coordinates, the background wind velocity is expressed as

$$
U=|\mathbf{U}| \cos \psi, \quad V=|\mathbf{U}| \sin \psi .
$$

These expressions must be differentiated and multiplied in various ways, applied at the surface, where $|\mathbf{U}|=\left|\mathbf{U}_{0}\right|$ and $\psi=\psi_{0}$, and inserted into (31)-(32).

Additionally, following Phillips (1984), the relevant directions for the drag are that along the wind direction at the surface, $D$, and the one perpendicular 
to the right, $T$. These can be related to $D_{x}$ and $D_{y}$ through

$$
D=D_{x} \cos \psi+D_{y} \sin \psi, \quad T=D_{x} \sin \psi-D_{y} \cos \psi .
$$

The two components of the drag are thus found to be given by

$$
\begin{aligned}
& D=\rho_{0} N\left|\mathbf{U}_{0}\right| b h_{0}^{2} G\left[B \cos ^{2} \psi+C \sin ^{2} \psi-\frac{1}{8\left(1-\gamma^{2}\right)}\left(a_{1} \frac{\left|\mathbf{U}_{0}\right|^{\prime 2}}{N^{2}}\right.\right. \\
& \left.\left.+a_{2} \frac{\left|\mathbf{U}_{0}\right|^{2}\left(\psi_{0}^{\prime}\right)^{2}}{N^{2}}+a_{3} \frac{\left|\mathbf{U}_{0}\right|\left|\mathbf{U}_{0}\right|^{\prime} \psi_{0}^{\prime}}{N^{2}}+a_{4} \frac{\left|\mathbf{U}_{0}\right|\left|\mathbf{U}_{0}\right|^{\prime \prime}}{N^{2}}+a_{5} \frac{\left|\mathbf{U}_{0}\right|^{2} \psi_{0}^{\prime \prime}}{N^{2}}\right)\right], \\
& T=\rho_{0} N\left|\mathbf{U}_{0}\right| b h_{0}^{2} G\left[(B-C) \sin \psi_{0} \cos \psi_{0}-\frac{1}{8\left(1-\gamma^{2}\right)}\left(b_{1} \frac{\left|\mathbf{U}_{0}\right|^{\prime 2}}{N^{2}}\right.\right. \\
& \left.\left.+b_{2} \frac{\left|\mathbf{U}_{0}\right|^{2}\left(\psi_{0}^{\prime}\right)^{2}}{N^{2}}+b_{3} \frac{\left|\mathbf{U}_{0}\right|\left|\mathbf{U}_{0}\right|^{\prime} \psi_{0}^{\prime}}{N^{2}}+b_{4} \frac{\left|\mathbf{U}_{0}\right|\left|\mathbf{U}_{0}\right|^{\prime \prime}}{N^{2}}+b_{5} \frac{\left|\mathbf{U}_{0}\right|^{2} \psi_{0}^{\prime \prime}}{N^{2}}\right)\right],
\end{aligned}
$$

where

$$
\begin{aligned}
a_{1}= & (B-C)\left(\cos ^{4} \psi_{0}+\gamma^{2} \sin ^{4} \psi_{0}\right)+6\left(C-\gamma^{2} B\right) \sin ^{2} \psi_{0} \cos ^{2} \psi_{0} \\
a_{2}= & {\left[3 C-\left(2+\gamma^{2}\right) B\right] \cos ^{4} \psi_{0}+\left[\left(1+2 \gamma^{2}\right) C-3 \gamma^{2} B\right] \sin ^{4} \psi_{0} } \\
& +\left[\left(1+17 \gamma^{2}\right) B-\left(17+\gamma^{2}\right) C\right] \sin ^{2} \psi_{0} \cos ^{2} \psi_{0} \\
a_{3}= & {\left[24 \gamma^{2} B-\left(18+6 \gamma^{2}\right) C\right] \cos \psi_{0} \sin ^{3} \psi_{0} } \\
& +\left[24 C-\left(6+18 \gamma^{2}\right) B\right] \sin \psi_{0} \cos ^{3} \psi_{0} \\
a_{4}= & 2(B-C)\left(\cos ^{4} \psi_{0}+\gamma^{2} \sin ^{4} \psi_{0}\right)+12\left(C-\gamma^{2} B\right) \sin ^{2} \psi_{0} \cos ^{2} \psi_{0} \\
a_{5}= & {\left[8 \gamma^{2} B-\left(6+2 \gamma^{2}\right) C\right] \cos \psi_{0} \sin ^{3} \psi_{0} } \\
& +\left[8 C-\left(2+6 \gamma^{2}\right) B\right] \sin \psi_{0} \cos ^{3} \psi_{0} \\
b_{1}= & {\left[\left(3+\gamma^{2}\right) C-4 \gamma^{2} B\right) \cos \psi_{0} \sin ^{3} \psi_{0} } \\
& +\left[\left(1+3 \gamma^{2}\right) B-4 C\right] \sin \psi_{0} \cos ^{3} \psi_{0} \\
b_{2}= & {\left[\left(1+11 \gamma^{2}\right) B-\left(10+2 \gamma^{2}\right) C\right] \cos _{0} \sin ^{3} \psi_{0} } \\
& +\left[\left(11+\gamma^{2}\right) C-\left(2+10 \gamma^{2}\right) B\right] \sin _{0} \cos ^{3} \psi_{0} \\
b_{3}= & {\left[\left(30+6 \gamma^{2}\right) C-\left(6+30 \gamma^{2}\right) B\right] \sin ^{2} \psi_{0} \cos ^{2} \psi_{0} } \\
& -6\left(C-\gamma^{2} B\right)\left(\sin ^{4} \psi_{0}+\cos ^{4} \psi_{0}\right) \\
b_{4}= & {\left[\left(6+2 \gamma^{2}\right) C-8 \gamma^{2} B\right] \cos \psi_{0} \sin ^{3} \psi_{0} } \\
& +\left[\left(2+6 \gamma^{2}\right) B-8 C\right] \sin \psi_{0} \cos ^{3} \psi_{0} \\
b_{5}= & {\left[\left(10+2 \gamma^{2}\right) C-\left(2+10 \gamma^{2}\right) B\right] \sin ^{2} \psi_{0} \cos ^{2} \psi_{0} } \\
& -2\left(C-\gamma^{2} B\right)\left(\sin ^{4} \psi_{0}+\cos ^{4} \psi_{0}\right)
\end{aligned}
$$

Equations (B.3)-(B.4) may also be normalised by the corresponding zerothorder drag (cf. Phillips 1984),

$$
\begin{aligned}
D_{0} & =\rho_{0} N\left|\mathbf{U}_{0}\right| b h_{0}^{2} G\left(B \cos ^{2} \psi_{0}+C \sin ^{2} \psi_{0}\right), \\
T_{0} & =\rho_{0} N\left|\mathbf{U}_{0}\right| b h_{0}^{2} G(B-C) \sin \psi_{0} \cos \psi_{0},
\end{aligned}
$$

as was done in Cartesian coordinates, and the resulting expressions naturally do not depend on $G$, being therefore valid for any orography with an elliptical horizontal cross-section. 


\section{ACKNOWLEDGEMENTS}

We thank Tor Häkon Sivertsen for instructive conversations, and also for providing a copy of his MSc thesis and other papers on mountain waves. This work was supported by Fundação para a Ciência e Tecnologia (FCT) under project BOSS Contract POCTI/CTE-ATM/58932/2004, co-financed by the European Union under program FEDER. MACT acknowledges the financial support of FCT under grant SFRH/BPD/3533/2000.

Baines, P. G. and Palmer, T. N. 1990

Bender, C. M. and Orszag, S. A. 1999

Gill, A. E

Grisogono, B.

Grubišić, V. and Smolarkiewicz, P. K.

Keller, T. L.

Lott, F. and Miller, M. J.

Miranda, P. M. A. and James, I. 1992 N.

Phillips, D. S.

Shutts, G.

Shutts, G. J. and Gadian, A.

Sivertsen, T. H.

Smith, R. B.

Smith, R. B.

Smith, R. B.

Teixeira, M. A. C. and Miranda, P. M. A.

Teixeira, M. A. C., Miranda, P. 2004 M. A. and Valente, M. A.

Teixeira, M. A. C. and Miranda, 2005 P. M. A.

Teixeira, M. A. C., Miranda, P. 2005 M. A., Argain, J. L. and Valente, M. A.

Valente, M. A.

Vosper, S. B.

1995

1999

1972

1979

1980

2000

1995

\section{REFERENCES}

Rationale for a new physically based parametrization of subgrid scale orographic effects. Tech. Memo. 169. European Centre for Medium-Range Weather Forecasts

Advanced Mathematical Methods for Scientists and Engineers, Springer

1982 Atmosphere-Ocean Dynamics, Academic Press

1994 Dissipation of wave drag in the atmospheric boundary layer. J. Atmos. Sci., 51, 1237-1243

The effect of critical levels on 3D orographic flows: linear regime. J. Atmos. Sci., 54, 1943-1960

Implications of the hydrostatic assumption on atmospheric gravity waves. J. Atmos. Sci., 51, 1915-1929

A new subrid-scale orographic drag parametrization: its formulation and testing. Quart. J. Roy. Meteor. Soc., 123, 101-127

Non-linear three dimensional effects on the wave drag: splitting flow and breaking waves. Quart. J. Roy. Meteor. Soc., 118, 1057-1081

1984 Analytical surface pressure and drag for linear hydrostatic flow over three-dimensional elliptical mountains. $J$. Atmos. Sci., 41, 1073-1084

Gravity-wave drag parametrization over complex terrain: The effect of critical-level absorption in directional wind-shear. Quart. J. Roy. Meteor. Soc., 121, 10051021

Numerical simulation of orographic gravity waves in flows which back with height. Quart. J. Roy. Meteor. Soc., 125, 2743-2765

Om Stasjonære Fjellbølger. MSc thesis (in Norwegian), University of Oslo, $119 \mathrm{pp}$.

The influence of the Earth's rotation on mountain wave drag. J. Atmos. Sci., 36, 177-180

Linear theory of stratified hydrostatic flow past an isolated obstacle. Tellus, 32, 348-364

Further development of a theory of lee cyclogenesis. $J$. Atmos. Sci., 43, 1582-1602

The effect of wind shear and curvature on gravity wave drag produced by a ridge. J. Atmos. Sci., 61, 2638-2643

An analytical model of mountain wave drag for wind profiles with shear and curvature. J. Atmos. Sci., 61, 1040-1054

Linear criteria for wave breaking in resonant stratified flow over a ridge. Quart. J. Roy. Meteor. Soc., 131, 18151820

Resonant gravity wave drag enhancement in linear stratified flow over mountains. Quart. J. Roy. Meteor. Soc., 131, $1795-1814$

Effects of directional wind shear on orographic gravity wave drag. PhD Thesis, University of Reading, 140 pp.
Observations and modelling of orographic internal gravity waves. PhD thesis, University of Leeds, $286 \mathrm{pp}$. 